\title{
A Single-Step Process for Preparing Supercapacitor Electrodes from Carbon Nanotubes
}

\author{
Rui Shi ${ }^{1}$, Lu Jiang ${ }^{1}$, Chunxu Pan ${ }^{1,2^{*}}$ \\ ${ }^{1}$ School of Physics and Technology and Key Laboratory of Artificial Micro-and Nano-structures of Ministry of Education, Wuhan \\ University, Wuhan, China; ${ }^{2}$ Center for Electron Microscopy, Wuhan University, China \\ Email: cxpan@whu.edu.cn
}

Received January $10^{\text {th }}, 2011$; revised January $20^{\text {th }}$, 2011; accepted January $24^{\text {th }}, 2011$.

\begin{abstract}
This paper introduces an easy single-step process for preparing the supercapacitor electrode from carbon nanotubes (CNTs) which were directly grown on a nickel foam framework by a chemical vapor deposition (CVD) technique. Due to the hierarchical porous structure and robust CNT-metal contacts, the present electrode exhibits better capacitive performance and lower internal resistance than the regular transfer method. Its specific capacitance of $25 \mathrm{~F} / \mathrm{g}$ is $127 \%$ higher than the value of the electrode prepared by the transfer method. This work proposes a simple, effective and economical approach for preparing a supercapacitor electrode without additional catalysts and pre- or post-treatments.
\end{abstract}

Keywords: Carbon Nanotubes, Supercapacitor, Energy Storage and Conversion

\section{Introduction}

Supercapacitors, also known as electric double layer capacitors or electrochemical capacitors, store energy using reversible ion adsorption on the surface of highly porous materials. They are very promising to be applied as longlife and high-power energy storage devices [1]. Carbon nanotubes (CNTs) have been recognized as a kind of attractive electrode materials for supercapacitors because of its high accessible surface area, low electric resistance and interconnected network structure [2,3]. Instead of attaching CNTs to the current collectors mechanically (the transfer method) [2,4,5], the method of directly growing CNTs on current collectors has received considerable attentions due to the robust CNT-metal contacts and one-step process [6]. Recently, Many efforts have been made for directly growing CNTs on bulk substrates, such as $\mathrm{Al}$ [7], Ni [8], Ta [9], stainless steel [10] and Inconel [6,11], achieving desired electrochemical capacitive properties. However, these direct growth processes generally use a chemical vapor deposition (CVD) technique through catalyst based methods [6,7]. Therefore, the complex substrate pretreatments are required including coating an $\mathrm{Al}_{2} \mathrm{O}_{3}$ film using electron beam evaporation $[9,11]$ and multistage processes using plasma enhanced techniques $[8,10]$. Furthermore, by using simple two-dimensional bulk substrates, only a lim- ited exterior surface of the metal substrate is utilized. Recently, J. R. McDonough et al. [12] showed that supercapacitors based on carbon nanofibers grown on porous nickel foam substrates had large per-area capacitances. However, in this approach, the substrate pretreatment with atomic layer deposition was required to help the nucleation and growth of carbon nanofibers.

In this letter, we present a direct growth approach of CNTs on a three-dimensional porous nickel (Ni) foam for fabricating supercapacitor electrodes by using an easy single-step CVD technique without any additional catalysts and pre-or post-treatments. Owing to the hierarchical porous structure and robust CNT-metal contacts, we demonstrate that the present electrode exhibits higher electrochemical capacitance, lower internal resistance and superior capacitive performance at very high current densities than the regular transfer method.

\section{Materials and Methods}

The CNTs were synthesized directly on a $\mathrm{Ni}$ foam in a quartz tube furnace CVD system using an easy singlestep approach. The Ni foam on a quartz boat was placed in a quartz tube and $200 \mathrm{sccm}$ Ar was continuously introduced into the quartz tube to exhaust air. The CNTs were grown on the porous Ni foam substrate by blowing $30 \mathrm{sccm} \mathrm{C}_{2} \mathrm{H}_{2}$ in the atmosphere of $\mathrm{Ar}$ at $610^{\circ} \mathrm{C}$ inside the quartz tube for $10 \mathrm{~min}$. 
The grown CNTs on Ni foam framework (Ni foamCNTs) were directly used as an electrode for making supercapacitors. For comparison, the transfer method was also used to fabricate the CNT electrodes (transfer CNTs). That is, the CNTs were removed from the Ni foam, and were prepared as pellets by pressing a mixture of $91 \mathrm{wt} \%$ of as-grown CNTs and 9 wt\% of polytetrafluoroethene (PTFE) binder. The total mass of active electrode materials was used to calculate specific capacitance.

The microstructure was observed by using a transmission electron microscope (TEM) (JEOL JEM 2010, Japan). The electrodes were characterized by a scanning electron microscope (SEM) (SIRION, FEI, the Netherlands). Electrochemical characterizations were carried out by an electrochemical workstation (CHI 660C, China) in $6 \mathrm{M} \mathrm{KOH}$ aqueous solution at room temperature in a three electrode configuration using a saturated calomel electrode (SCE) as the reference electrode. Different electrochemical techniques were used to characterize the capacitive performance, and internal resistance of supercapacitor electrodes. Cyclic voltammetry $(\mathrm{CV})$ were performed between -0.9 and $-0.1 \mathrm{~V}$ at different scan rates. A galvanostatic charge-discharge experiment was carried out between -0.9 and $-0.1 \mathrm{~V}$ at the current density range from 0.25 to $5 \mathrm{~A} / \mathrm{g}$. The electrochemical impedance spectroscopy at a dc bias of $-0.9 \mathrm{~V}$ was performed with a 10 $\mathrm{mV}$ AC signal amplitude in the frequency range from 100 $\mathrm{kHz}$ to $10 \mathrm{mHz}$.

\section{Results and Discussion}

Figure 1 and Figure 2 show the SEM and TEM images of CNTs directly grown on porous $\mathrm{Ni}$ foam, respectively. Obviously, the entangled CNTs were well grown on the surface of $\mathrm{Ni}$ foam framework. Comparing with the CNTs grown on bulk substrates, the present CNTs on the porous substrate make full use of the large internal surface area of the $\mathrm{Ni}$ foam. A hierarchical porous structure is therefore formed. That is, the $\mathrm{Ni}$ foam framework provides macropores which act as transport channels into the interior pores of the electrode. The interconnected CNTs network produces mesopores and micropores at the interstices, surface and central canals of the intertwined CNTs which contributes dominantly to the capacitance of supercapacitor electrodes [13]. The CNT-Ni foam interface of electrode fabricated by using the transfer method is present in Figure 3. The CNTs were aggregated and indirectly mechanically attached to the Ni foam framework mechaniccally via binder. There are obvious spatial gaps between CNTs and $\mathrm{Ni}$ foam framework which block the electronic transportation at this interface and therefore increase the internal resistance of supercapacitor electrodes.
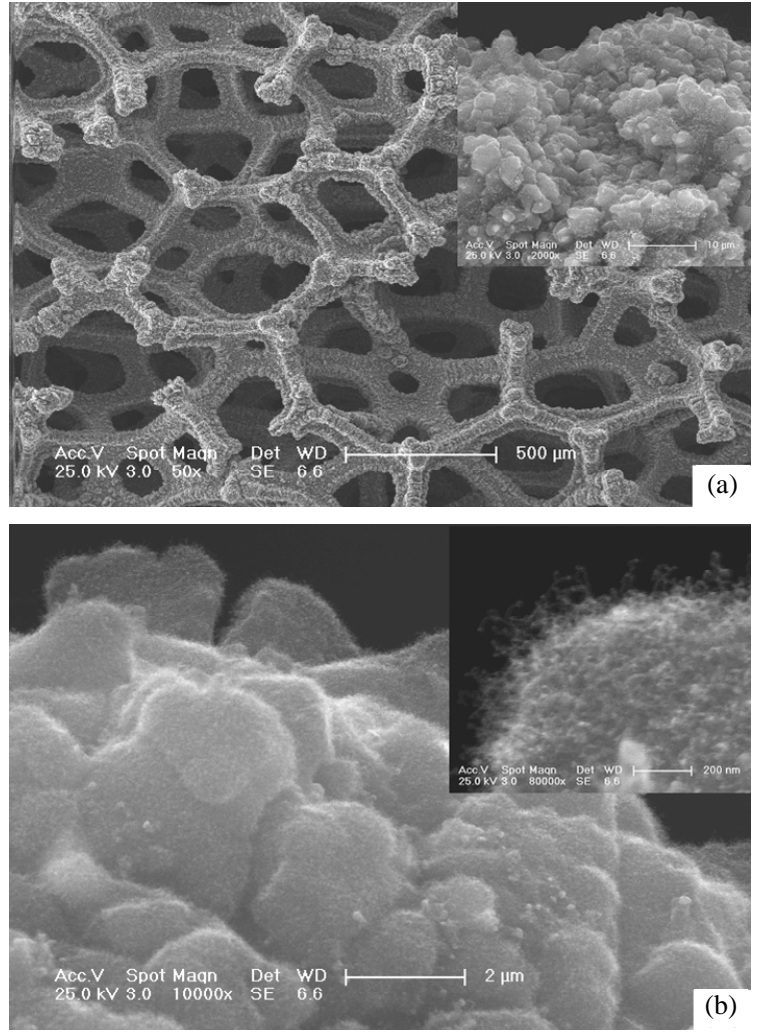

Figure 1. SEM morphologies of CNTs grown on the Ni foam. (a) Low magnification; (b) High magnification.

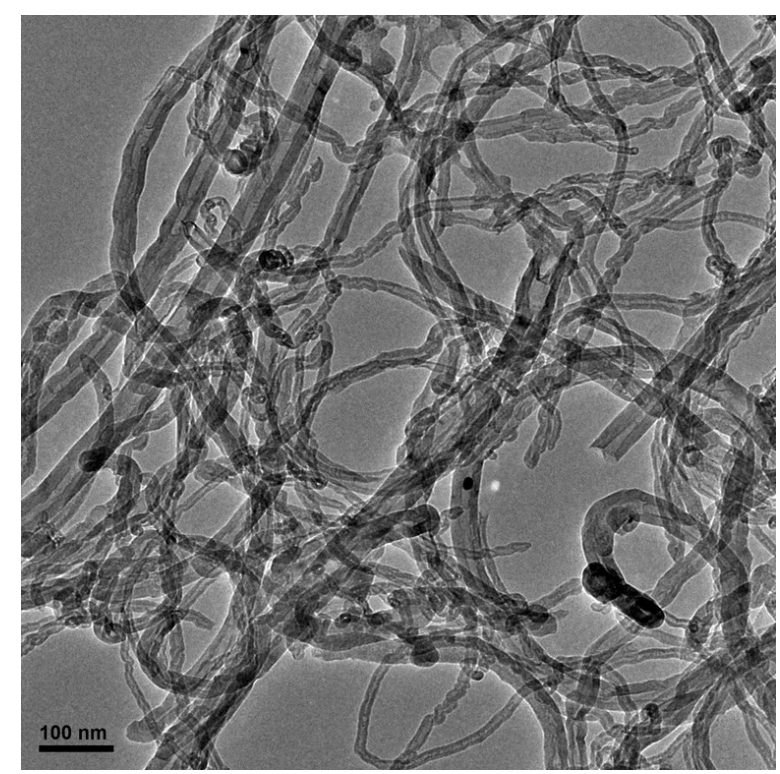

Figure 2. TEM image of CNTs grown on the Ni foam.

Figure 4(a) illustrates the CV curves of the Ni foamCNTs and the transfer CNTs. Both electrodes present regular, rectangular shapes at a relatively high scan rate (200 mV/s), indicating well-developed capacitive properties [2]. However, the electrode with Ni foam-CNTs ex- 

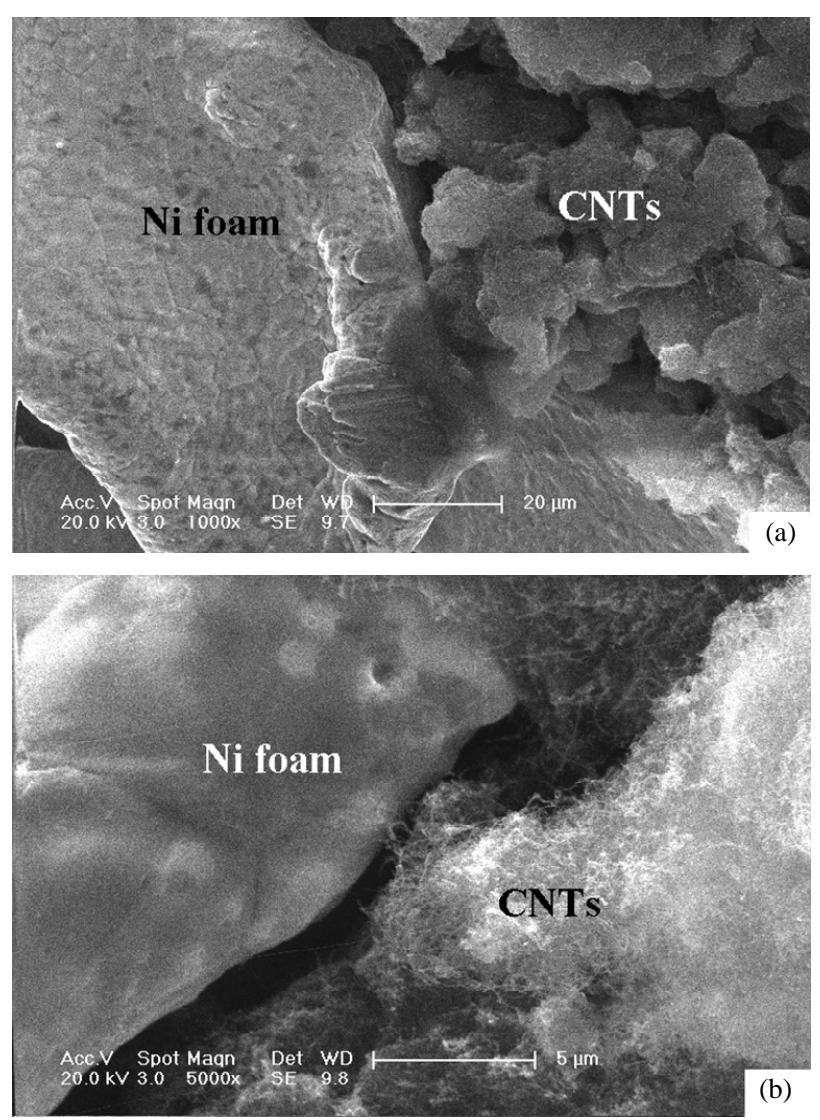

Figure 3. SEM morphologies of CNT-Ni foam interface of electrode fabricated by using the transfer method. (a) low magnification; (b) high magnification.

hibits much higher specific capacitance, because its $\mathrm{CV}$ curve covers a much larger area. Figure 4(b) is the ratio of retained capacitance calculated from galvanostatic charge-discharge experiments of both electrodes as a function of charge-discharge current density. As the current density rises to a very high value of $5 \mathrm{~A} / \mathrm{g}$, the specific capacitance of $\mathrm{Ni}$ foam-CNTs retains a value of $72 \%$ at the current density of $0.25 \mathrm{~A} / \mathrm{g}$, which exhibits an impressive capacitive performance at high current densities. However, a CNT electrode prepared by the transfer method loses $67 \%$ of capacitance at the same current density.

The Nyquist plots (Figure 4(c)) show an inconspicuous semi-circle at high frequencies and a steeper slope at low frequencies for the electrode with Ni foam-CNTs, which indicates that the Ni foam-CNTs electrode exhibits much lower internal resistance than that of the CNT electrode prepared by the transfer method [14,15]. In order to confirm these results, the comparison of the IR drop for both electrodes at different charge-discharge currents was preceded, as shown in Figure 4(d). Each branch of the galvanostatic charge-discharge curves starts with a volt-
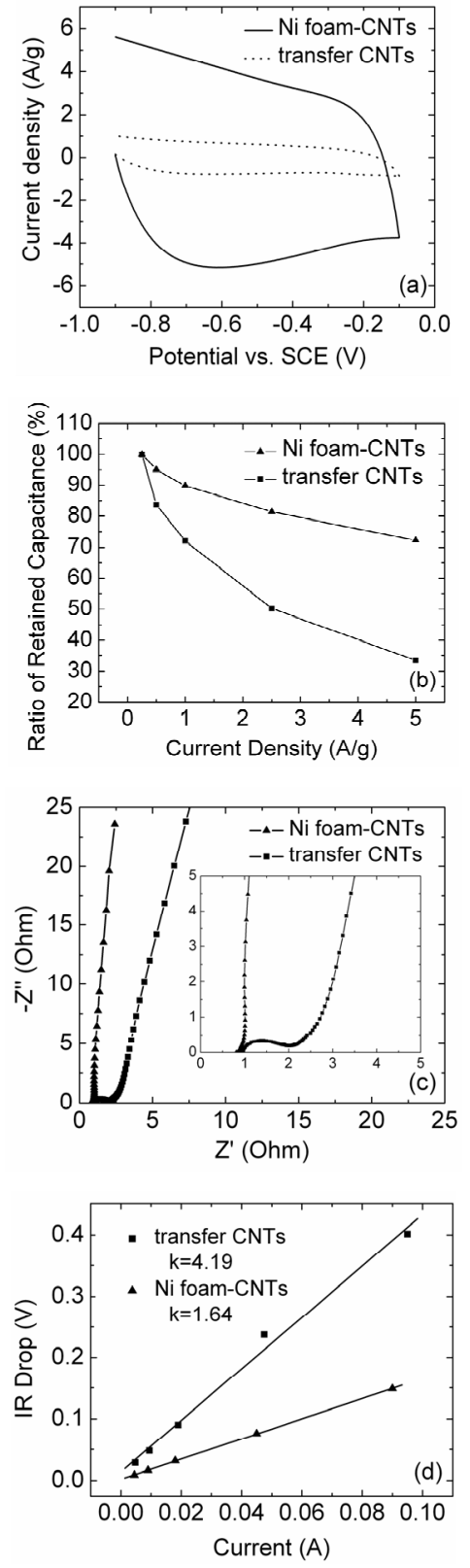

Figure 4. (a) CV curves taken at $200 \mathrm{mV} / \mathrm{s}$; (b) The ratio of retained capacitance values as a function of charge-discharge current density; (c) Nyquist plots $(0.01 \sim 100000 \mathrm{~Hz})$. The upper inset is a local amplified image at high frequencies; (d) IR drop plots of the Ni foam-CNTs and transfer CNTs electrodes.

age drop (IR drop) due to the internal resistance of electrode [16], and the slope of the linear relationship between the IR drop and charge-discharge current can be used to estimate the over-all resistance [15]. From Figure 4(d), the slope $\mathrm{k}$ is 1.64 and 4.19 for the Ni foam-CNTs and the transfer CNTs respectively, which reveals a better conductivity of electrode built from the CNTs grown on 
Table 1. Specific capacitances of the supercapacitor electrodes prepared using different techniques.

\begin{tabular}{ccccc}
\hline electrode material & $\begin{array}{c}\text { cyclic voltammetry } \\
(\mathrm{F} / \mathrm{g})\end{array}$ & $\begin{array}{c}\text { galvanostatic charge-discharge } \\
(\mathrm{F} / \mathrm{g})\end{array}$ & $\begin{array}{c}\text { electrochemical impedance } \\
\text { spectroscopy (F/g) }\end{array}$ & $\begin{array}{c}\text { average specific } \\
\text { capacitance (F/g) }\end{array}$ \\
\hline Ni foam-CNTs & 25 & 24 & 25 & 25 \\
Transfer CNTs & 9 & 13 & 10 & 11 \\
\hline
\end{tabular}

Ni foam.

The above experimental results indicate that the electrode with the Ni foam-CNTs has a significant higher specific capacitance than that of the CNT electrode prepared by using the transfer method. This can be mainly attributed to the hierarchical porous structure of $\mathrm{Ni}$ foam-CNTs electrode, which facilitates the charging and discharging of electric double-layer by profiting the penetration of ion at the electrode/electrolyte interface through a proper pore-size distribution [13]. The average specific capacitance of the $\mathrm{Ni}$ foam-CNTs electrode measured using three electrochemical techniques is 25 F/g as given in Table 1, which is $127 \%$ higher than the value of the CNT electrode prepared by the transfer method at the same conditions. At high charge-discharge current densities, easily permeable hierarchical porous structure also contributes to the impressive capacitive performance of the $\mathrm{Ni}$ foam-CNTs electrode by making better use of electrochemically accessible pores. The better conductivity of the Ni foam-CNTs electrode owes to the robust CNT-metal contacts-another important factor for determining the excellent capacitive performance at high current densities. For the CNTs electrode prepared by the transfer method, the high internal resistance can increase the voltage drop and resistive energy loss, which will restrain the capacitive performance of electrode and this effect, is enhanced remarkably at high charge-discharge current densities.

\section{Conclusions}

In conclusion, the present process provides an effective approach for directly preparing a supercapacitor electrode from CNTs without any additional catalysts and pre- or post-treatments. It presents much higher electrochemical capacitance, lower internal resistance and better capacitive performance at very high current densities than the CNT electrode prepared by the conventional transfer method. It is expected to have wide applications in various fields such as chemical and biological sensors and catalyst supports.

\section{Acknowledgements}

This work was supported by the National Innovative Experimental Program for Undergraduates, Ministry of Education of China (No. 061048608), and the National Nature Science Foundation of China (No. J0830310).

\section{REFERENCES}

[1] R. Kotz and M. Carlen, "Principles and Applications of Electrochemical Capacitors,” Electrochimica Acta, Vol. 45, No. 15, May 2000, pp. 2483-2498. doi:10.1016/S0013-4686(00)00354-6

[2] E. Frackowiak, K. Metenier, V. Bertagna and F. Beguin, "Supercapacitor Electrodes from Multiwalled Carbon Nanotubes," Applied Physics Letters, Vol. 77, No. 15, October 2000, pp. 2421-2423.

doi:10.1063/1.1290146

[3] C. Niu, E. K. Sichel, R. Hoch, D. Moy and H. Tennent, "High Power Electrochemical Capacitors Based on Carbon Nanotube Electrodes,” Applied Physics Letters, Vol. 70, No. 11, March 1997, pp. 1480-1482. doi:10.1063/1.118568

[4] H. Zhang, G. P. Cao and Y. S. Yang, "Using a Cut-Paste Method to Prepare a Carbon Nanotube Fur Electrode," Nanotechnology, Vol. 18, No. 19, May 2007, p. 195607. doi:10.1088/0957-4484/18/19/195607

[5] C. G. Liu, M. Liu, F. Li and H. M. Cheng, "Frequency Response Characteristic of Single-Walled Carbon Nanotubes as Supercapacitor Electrode Material,” Applied Physics Letters, Vol. 92, No. 14, April 2008, pp. 143108(3).

[6] S. Talapatra, S. Kar, S. K. Pal, R. Vajtai, L. Ci, P. Victor, M. M. Shaijumon, S. Kaur, O. Nalamasu and P. M. Ajayan, "Direct growth of aligned carbon nanotubes on bulk metals," Nature Nanotechnology, Vol. 1, No. 2, November 2006, pp. 112-116. doi:10.1038/nnano.2006.56

[7] C. Emmenegger, P. Mauron, A. Zuttel, C. Nutzenadel, A. Schneuwly, R. Gallay and L. Schlapbach, "Carbon Nanotube Synthesized on Metallic Substrates," Applied Surface Science, Vol. 162-163, No. 1, August 2000, pp. 452-456. doi:10.1016/S0169-4332(00)00232-4

[8] B. J. Yoon, S. H. Jeong, K. H. Lee, H. S. Kim, C. G. Park and J. H. Han, "Electrical Properties of Electrical Double layer Capacitors with Integrated Carbon Nanotube Electrodes," Chemical Physics Letters, Vol. 388, No. 1-3, April 2004, pp. 170-174. doi:10.1016/j.cplett.2004.02.071

[9] H. Zhang, G. P. Cao, Z. Y. Wang, Y. S. Yang and Z. N. $\mathrm{Gu}$, "Electrochemical Capacitive Properties of Carbon Nanotube Arrays Directly Grown on Glassy Carbon and Tantalum Foils," Carbon, Vol. 46, No. 5, April 2008, pp. 822-824. doi:10.1016/j.carbon.2008.02.015

[10] D. Park, Y. H. Kim and J. K. Lee, "Synthesis of Carbon Nanotubes on Metallic Substrates by a Sequential Com- 
bination of PECVD and Thermal CVD," Carbon, Vol. 41, No. 5, April 2003, pp. 1025-1029. doi:10.1016/S0008-6223(02)00432-3

[11] L. J. Gao, A. P. Peng, Z. Y. Wang, H. Zhang, Z. J. Shi, Z. N. Gu, G. P. Cao and B. Z. Ding, "Growth of Aligned Carbon Nanotube Arrays on Metallic Substrate and Its Application to Supercapacitors," Solid State Communications, Vol. 146, No. 9-10, June 2008, pp. 380-383. doi:10.1016/j.ssc.2008.03.034

[12] J. R. McDonough, J. W. Choi, Y. Yang, F. L. Mantia, Y. G. Zhang and Y. Cui, "Carbon Nanofiber Supercapacitors with Large Areal Capacitances,” Applied Physics Letters, Vol. 95, No. 24, December 2009, pp. 243109(3).

[13] A. G. Pandolfo and A. F. Hollenkamp, "Carbon Properties and Their Role in Supercapacitors," Journal of Power Sources, Vol 157, No. 1, June 2006, pp. 11-27. doi:10.1016/j.jpowsour.2006.02.065
[14] M. Toupin, D. Belanger, I. R. Hill and D. Quinn, "Performance of Experimental Carbon Blacks in Aqueous Supercapacitors,” Journal of Power Sources, Vol. 140, No. 1, January 2005, pp. 203-210. doi:10.1016/j.jpowsour.2004.08.014

[15] Y. M .Tian, Y. Song, Z. H. Tang, Q. G. Gui and L. Liu, "Influence of High Temperature Treatment of Porous Carbon on the Electrochemical Performance in Supercapacitor," Journal of Power Sources, Vol. 184, No. 2, October 2008, pp. 675-681. doi:10.1016/j.jpowsour.2008.04.070

[16] M. Kaempgen, J. Ma, G. Gruner, G. Wee and S. G. Mhaisalkar, "Bifunctional Carbon Nanotube Networks for Supercapacitors,” Applied Physics Letters, Vol. 90, No. 27, June 2007, p. 264104. doi:10.1063/1.2749187 